\title{
Sinc Nyström method for a class of nonlinear Volterra integral equations of the first kind
}

\author{
Yanying Ma', Jin Huang ${ }^{1}$, Changqing Wang ${ }^{2}$ and $\mathrm{Hu} \mathrm{Li}^{1}$
}

\author{
"Correspondence: \\ ma_yan_ying@126.com \\ 'School of Mathematical Sciences, \\ University of Electronic Science and \\ Technology of China, Chengdu, \\ Sichuan 611731, P.R. China \\ Full list of author information is \\ available at the end of the article
}

\begin{abstract}
Two numerical methods are proposed to solve nonlinear Volterra integral equations of the first kind. By using variable transformations, the problem is converted into linear Volterra integral equations of the second kind. These methods are implemented by utilizing Sinc quadrature, and then the problem is reduced to linear algebraic system equations. We state error analysis for the proposed methods, which show that these methods obtain exponential convergence order. Numerical examples are presented to confirm the theoretical estimation and illustrate the effectiveness of the proposed methods.
\end{abstract}

Keywords: nonlinear Volterra integral equation; Sinc Nyström method; SE transformation; DE transformation

\section{Introduction}

Volterra integral equations of the first kind arise in many fields of science and engineering, for example, in diffusion problems, fluid dynamics, heat conduction problems, nonlinear dynamic systems identification, concrete problems of mechanics, et cetera. As we all know, Volterra integral equations of the first kind are ill-posed problems because their solutions are generally unstable, and slight changes can make large errors [1,2]. So it is difficult to find exact solutions of these equations in many cases. Furthermore, since the small error may lead to an unbounded error, it is also difficult to find numerical solutions. Some works were motivated by the aforementioned discussion, and several regularization methods were introduced to conquer the ill-posedness in [3-5].

This paper is focused on proposing two numerical methods for solving a class of nonlinear Volterra integral equations of the first kind in the form

$$
\int_{a}^{x} K(x, t) H(u(t)) d t=f(x), \quad x \in[a, b]
$$

where $K, H$, and $f$ are given functions, $H$ is invertible, and $u$ is the solution to be determined under the condition $f(a)=0$.

There exist several methods to solve linear Volterra integral equations of the first kind [6-9], such as block-pulse functions method [6], modified block-pulse functions method

(c) $2016 \mathrm{Ma}$ et al. This article is distributed under the terms of the Creative Commons Attribution 4.0 International License (http://creativecommons.org/licenses/by/4.0/), which permits unrestricted use, distribution, and reproduction in any medium, provided you give appropriate credit to the original author(s) and the source, provide a link to the Creative Commons license, and indicate if changes were made. 
[7], and wavelet method [8]. However, nonlinear problems are still a challenge. Babolian et al. introduced the operational matrices method by using piecewise constant orthogonal functions and homotopy perturbation method for solving nonlinear Volterra integral equations of the first kind separately in [10] and [11]. The Adomian method [12] and optimal homotopy asymptotic method [13] were applied to solve nonlinear Volterra integral equations. Inderdeep and Sheo presented the Haar wavelet method for numerical solution of a class of nonlinear Volterra integral equations of the first kind in [14].

It is common to employ a collocation method or analytic method based on the use of polynomial base functions to solve Volterra integral equations. Recently, several authors introduced SE and DE Sinc quadratures for solving integral equations. Muhammad and Mori proposed a numerical method of indefinite integration based on the DE transformation together with Sinc expansion of the integrand in [15]. Haber provided two formulas and approximation error for approximating the indefinite integral over a finite interval in [16]. The Sinc Nyström method for numerical solution of one-dimensional Cauchy singular integral equations given on a smooth arc in the complex plane has been described in [17]. Muhammad et al. [18] presented a technique for linear integral equations using the Sinc collocation method based on the DE transformation. Rashidinia and Zarebnia [19] developed an analogous approach for the system of linear Fredholm integral equations by means of SE transformation. More recently, Okayama et al. [20] reported error estimates with explicit constants for the Sinc approximation, Sinc quadrature, and Sinc indefinite integration. Furthermore, the theoretical analysis of Sinc Nyström methods for linear integral and differential equations have been discussed in [21-23]. Similar numerical approaches for nonlinear Fredholm and Volterra integral equations of the second kind are also presented in $[24,25]$. However, nonlinear Volterra integral equations of the first kind are still not solved. In this work, we develop SE and DE Sinc methods to solve Eq. (1) in terms of SE and DE Sinc quadrature rules; these methods have a simple structure and perfect approximate properties. The convergence rates of these methods are exponential. Therefore, the proposed methods improve the conventional polynomial convergence rate. Furthermore, the proposed schemes are stable because the discrete coefficient matrices are very well conditioned.

In this paper, the basic ideas are organized as follows. In Section 2, we present some definitions and preliminary results about the Sinc function and SE, DE Sinc quadrature for indefinite integral. In Section 3, Sinc Nyström methods for the nonlinear Volterra integral equations of the first kind are developed. In Section 4, the convergence analysis with errors are described for the current methods. Both of these two algorithms are exponentially convergent. In Section 5, numerical examples are presented to validate the effectiveness of these methods. Numerical results of the proposed methods are compared with existing methods to confirm the reliability of the proposed methods. Finally, a conclusion is given in Section 6.

\section{Preliminaries}

\subsection{Sinc indefinite integral on the real axis}

In this section, we give a summary of the basic formulation of the Sinc function [26]. We introduce some known results and useful formulas. The Sinc function is defined on the 
whole real line by

$$
\operatorname{Sinc}(x)= \begin{cases}\frac{\sin (\pi x)}{\pi x}, & x \neq 0 \\ 1, & x=0\end{cases}
$$

The Sinc approximation for a function $f$ on the entire real axis can be expressed in the truncated sum form

$$
f(x) \approx \sum_{i=-N}^{N} f(i h) S(i, h)(x), \quad x \in \mathbb{R},
$$

where the basis function $S(i, h)(x)=\frac{\sin [\pi(x / h-i)]}{\pi(x / h-i)}$, and $h$ is a step size appropriately selected hinging on $N \in \mathbb{Z}^{+}$and $i=-N, \ldots, N$. Haber [16] introduced the numerical indefinite integral formula by employing the Sinc function as follows:

$$
\begin{aligned}
\int_{-\infty}^{s} f(x) d x & \approx \sum_{i=-N}^{N} f(i h) \int_{-\infty}^{s} S(i, h)(x) d x \\
& =\sum_{i=-N}^{N} f(i h) J(i, h)(s), \quad s \in \mathbb{R},
\end{aligned}
$$

where the basis function $J(i, h)$ is expressed as

$$
J(i, h)(s)=h\left\{\frac{1}{2}+\frac{1}{\pi} S i[\pi(s / h-i)]\right\}
$$

with $\operatorname{Si}(s)=\int_{0}^{s} \frac{\sin \mu}{\mu} d \mu$.

\subsection{SE and DE Sinc indefinite integral}

From the above we can see that the approximation of Eq. (4) is valid on $\mathbb{R}$, whereas Eq. (1) is defined on finite interval $[a, x]$. Equation (4) can be applicable to infinite intervals using variable transformations. Here, the smoothing variable transformations with standard SE and DE transformation functions $\phi(x)$ are utilized.

The SE transformation and its inverse can be presented as follows:

$$
\begin{aligned}
& \phi^{\mathrm{SE}}(x)=\frac{b-a}{2} \tanh \left(\frac{x}{2}\right)+\frac{b+a}{2}, \quad x \in \mathbb{R}, \\
& \left\{\phi^{\mathrm{SE}}\right\}^{-1}(t)=\log \left(\frac{t-a}{b-t}\right), \quad t \in(a, b) .
\end{aligned}
$$

In order to define a facilitate function space, we introduce the strip domain $\mathscr{D}_{d}=\{z \in \mathcal{C}$ : $|\operatorname{Im} z|<d\}$ for some $d>0$. The SE transformation maps $(a, b)$ onto $\mathbb{R}$ and maps $\mathscr{D}_{d}$ onto the region

$$
\phi^{\mathrm{SE}}\left(\mathscr{D}_{d}\right)=\left\{z \in \mathcal{C}\left|\arg \left(\frac{z-a}{b-z}\right)\right|<d\right\} .
$$

In order to obtain the results precisely, we need to introduce a number of spaces of functions analytic as follows. 
Definition 2.1 Let $\mathscr{D}$ be a simply connected domain satisfying $(a, b) \subset \mathscr{D}_{d}$, and let $\alpha$ be a positive constant. Then, $L_{\alpha}\left(\mathscr{D}_{d}\right)$ denotes the family of all functions $f$ satisfying the following conditions:

(i) $f$ is analytic in $\mathscr{D}_{d}$;

(ii) $|f(z)| \leq M_{0}|Q(z)|^{\alpha}$ for all $z$ in $\mathscr{D}_{d}$, where $Q(z)=(z-a)(b-z)$, and $M_{0}$ is a constant.

Based on the Sinc approximation and SE transformation, we can implement a quadrature rule designated as the SE Sinc quadrature and present exponential convergence in the following theorem.

Theorem 2.1 (see [20]) Assume that $(f Q) \in L_{\alpha}\left(\phi^{\mathrm{SE}}\right)$ with $0<d<\pi$ and let $h$ be selected by the formula

$$
h=\sqrt{\frac{\pi d}{\alpha N}}
$$

where $N \in \mathbb{Z}^{+}$. Then, there exists a constant $C_{\alpha}^{\text {SE }}$ such that

$$
\begin{aligned}
& \left|\int_{a}^{x} f(t) d t-\sum_{i=-N}^{N} f\left(\phi^{\mathrm{SE}}(i h)\right)\left\{\phi^{\mathrm{SE}}\right\}^{\prime}(i h) J(i, h)\left(\left\{\phi^{\mathrm{SE}}\right\}^{-1}(x)\right)\right| \\
& \quad \leq M_{0}(b-a)^{2 \alpha-1} C_{\alpha}^{\mathrm{SE}} e^{-\sqrt{\pi d \alpha N}},
\end{aligned}
$$

where $M_{0}$ is the constant in Definition 2.1.

In order to improve the convergence speed, Muhammad substituted the DE transformation for the SE transformation. The DE transformation and its inverse are described as follows:

$$
\begin{aligned}
& \phi^{\mathrm{DE}}(x)=\frac{b-a}{2} \tanh \left(\frac{\pi}{2} \sinh (x)\right)+\frac{b+a}{2}, \quad x \in \mathbb{R}, \\
& \left\{\phi^{\mathrm{DE}}\right\}^{-1}(t)=\log \left[\frac{1}{\pi} \log \left(\frac{t-a}{b-t}\right)+\sqrt{1+\left\{\frac{1}{\pi} \log \left(\frac{t-a}{b-t}\right)\right\}^{2}}\right], \quad t \in(a, b) .
\end{aligned}
$$

The DE transformation maps $\mathscr{D}_{d}$ onto the domain

$$
\phi^{\mathrm{DE}}\left(\mathscr{D}_{d}\right)=\left\{z \in \mathcal{C}\left|\arg \left[\frac{1}{\pi} \log \left(\frac{t-a}{b-t}\right)+\sqrt{1+\left\{\frac{1}{\pi} \log \left(\frac{t-a}{b-t}\right)\right\}^{2}}\right]\right|<d\right\} .
$$

If we use the DE transformation instead of the SE transformation, then the DE Sinc quadrature for indefinite is obtained. The convergence order of error is improved in the next theorem.

Theorem 2.2 (see [20]) Assume that $(f Q) \in L_{\alpha}\left(\phi^{\mathrm{DE}}\right)$ with $0<d<\pi / 2$, and let $h$ be selected by the formula

$$
h=\frac{\log (2 d N / \alpha)}{N},
$$


where $N \in \mathbb{Z}^{+}$. Then, there exists a constant $C_{\alpha}^{\text {SE }}$ such that

$$
\begin{aligned}
& \left|\int_{a}^{x} f(t) d t-\sum_{i=-N}^{N} f\left(\phi^{\mathrm{DE}}(i h)\right)\left\{\phi^{\mathrm{DE}}\right\}^{\prime}(i h) J(i, h)\left(\left\{\phi_{\mathrm{DE}}\right\}^{-1}(x)\right)\right| \\
& \quad \leq M_{0}(b-a)^{2 \alpha-1} C_{\alpha}^{\mathrm{DE}} \frac{\log (2 d N / \alpha)}{N} e^{\frac{-\pi d N}{\log (2 d N / \alpha)}}
\end{aligned}
$$

where $M_{0}$ is the constant in Definition 2.1.

\section{Sinc Nyström method for Volterra integral equations}

In this part, we consider the numerical solution of Eq. (1). First, the nonlinear Volterra integral equation of first kind is transformed to a linear Volterra integral equation of the second kind by setting

$$
H(u(t))=v(t)
$$

in Eq. (1). Therefore, we obtain the linear Volterra integral equation of the form

$$
\int_{a}^{x} K(x, t) v(t) d t=f(x), \quad x \in[a, b] .
$$

Then, taking the derivative with respect to $x$ in both sides of the last equation, we get

$$
K(x, x) v(x)+\int_{a}^{x} \frac{\partial K(x, t)}{\partial x} v(t) d t=f^{\prime}(x), \quad x \in[a, b] .
$$

Assume that the function $K(x, x) \neq 0$, so that Eq. (13) can be converted into a Volterra integral equation of the second kind

$$
v(x)+\int_{a}^{x} K_{1}(x, t) v(t) d t=f_{1}(x), \quad x \in[a, b]
$$

where $K_{1}(x, t)=\frac{\partial K(x, t)}{\partial x} / K(x, x)$ and $f_{1}(x)=f^{\prime}(x) / K(x, x)$.

Further, the proposed numerical methods for Eq. (1) will be fully discussed in two subsections, where we state the SE Sinc Nyström method and DE Sinc Nyström method for efficient evaluation of Volterra integral equations.

\subsection{SE Sinc Nyström method}

According to Theorem 2.1, SE Sinc indefinite integration can be directly applied to the integral term (on the left-hand side of Eq. (14)): the integral can be accurately approximated as

$$
\begin{aligned}
\int_{a}^{x} K_{1}(x, t) v(t) d t & \approx K_{N}^{\mathrm{SE}} v(x) \\
& =h \sum_{i=-N}^{N} K_{1}\left(x, \zeta_{i}^{\mathrm{SE}}\right)\left\{\phi^{\mathrm{SE}}\right\}^{\prime}(i h) J(i, h)\left(\left\{\phi^{\mathrm{SE}}\right\}^{-1}(x)\right) v_{i}^{\mathrm{SE}}
\end{aligned}
$$

where $v_{i}^{\mathrm{SE}}$ denotes an approximate value of $v\left(\phi^{\mathrm{SE}}(i h)\right), \zeta_{i}^{\mathrm{SE}}=\phi^{\mathrm{SE}}(i h)$, and the mesh $h$ is chosen by formula (6). The Nyström method is exploited to find the approximate solution 
$v_{N}^{\mathrm{SE}}$ for Eq. (14) such that

$$
v_{N}^{\mathrm{SE}}(x)+h \sum_{i=-N}^{N} K_{1}\left(x, \zeta_{i}^{\mathrm{SE}}\right)\left\{\phi^{\mathrm{SE}}\right\}^{\prime}(i h) J(i, h)\left(\left\{\phi^{\mathrm{SE}}\right\}^{-1}(x)\right) v_{i}^{\mathrm{SE}}=f_{1}(x) .
$$

There are $2 N+1$ unknowns $v_{i}^{\mathrm{SE}}(i=-N, \ldots, N)$ to be determined in Eq. (16). In order to determine these unknowns, we select the Sinc points $\zeta_{j}^{\mathrm{SE}}=\phi^{\mathrm{SE}}(j h)$ as quadrature points. By taking $x=\zeta_{j}^{\mathrm{SE}}$ in Eq. (16), we eventually gain the following linear system of equations with unknowns $v_{j}^{\mathrm{SE}}(j=-N, \ldots, N)$ :

$$
v_{j}^{\mathrm{SE}}+h \sum_{i=-N}^{N} K_{1}\left(\zeta_{j}^{\mathrm{SE}}, \zeta_{i}^{\mathrm{SE}}\right)\left\{\phi^{\mathrm{SE}}\right\}^{\prime}(i h) J(i, h)(j h) v_{i}^{\mathrm{SE}}=f_{1}\left(\zeta_{j}^{\mathrm{SE}}\right) .
$$

Note that $\left\{\phi^{\mathrm{SE}}\right\}^{-1}\left(\zeta_{j}^{\mathrm{SE}}\right)=j h$, so $J(i, h)\left(\left\{\phi^{\mathrm{SE}}\right\}^{-1}\left(\zeta_{j}^{\mathrm{SE}}\right)\right)=J(i, h)(j h)$, and $h$ is given by formula (6). Equation (17) can be stated in operator form as follows:

$$
\left(I_{N}+K_{N}^{\mathrm{SE}}\right) v_{N}^{\mathrm{SE}}=f_{N}^{\mathrm{SE}}
$$

Then, the approximate solution $v_{N}^{\mathrm{SE}}(x)$ at an arbitrary point $x$ of Eq. (14) can be expressed as

$$
v_{N}^{\mathrm{SE}}(x)=f_{1}(x)-h \sum_{i=-N}^{N} K_{1}\left(x, \zeta_{i}^{\mathrm{SE}}\right)\left\{\phi^{\mathrm{SE}}\right\}^{\prime}(i h) J(i, h)\left(\left\{\phi^{\mathrm{SE}}\right\}^{-1}(x)\right) v_{i}^{\mathrm{SE}} .
$$

In fact, $v_{N}^{\mathrm{SE}}(x)$ is also an approximate solution of Eq. (12). Using relation (11), we obtain an approximate solution $u_{N}^{\mathrm{SE}}(x)$ of Eq. (1),

$$
u_{N}^{\mathrm{SE}}(x)=H^{-1}\left(v_{N}^{\mathrm{SE}}(x)\right), \quad x \in[a, b] .
$$

\subsection{DE Sinc Nyström method}

Similarly, DE Sinc indefinite integration can also be directly employed to the second term kernel integral (on the left-hand side of Eq. (14)); based on Theorem 2.2, we derive the discrete DE operator

$$
\begin{aligned}
\int_{a}^{x} K_{1}(x, t) v(t) d t & \approx K_{N}^{\mathrm{DE}} v(x) \\
& =h \sum_{i=-N}^{N} K_{1}\left(x, \zeta_{i}^{\mathrm{DE}}\right)\left\{\phi^{\mathrm{DE}}\right\}^{\prime}(i h) J(i, h)\left(\left\{\phi^{\mathrm{DE}}\right\}^{-1}(x)\right) v_{i}^{\mathrm{DE}}
\end{aligned}
$$

where $v_{i}^{\mathrm{DE}}$ represents an approximate value of $v\left(\phi^{\mathrm{DE}}(i h)\right), \zeta_{i}^{\mathrm{DE}}=\phi^{\mathrm{DE}}(i h)$, and the mesh $h$ is selected by (9). The Nyström method is utilized to obtain the $v_{N}^{\mathrm{DE}}$ such that

$$
v_{N}^{\mathrm{DE}}(x)+h \sum_{i=-N}^{N} K_{1}\left(x, \zeta_{i}^{\mathrm{DE}}\right)\left\{\phi^{\mathrm{DE}}\right\}^{\prime}(i h) J(i, h)\left(\left\{\phi^{\mathrm{DE}}\right\}^{-1}(x)\right) v_{i}^{\mathrm{DE}}=f_{1}(x) .
$$

There are $2 N+1$ unknowns $v_{i}^{\mathrm{DE}}(i=-N, \ldots, N)$ to be determined in Eq. (22). In order to determine these $2 N+1$ unknown values, we choose the Sinc points $\zeta_{j}^{\mathrm{DE}}=\phi^{\mathrm{DE}}(j h)$ as 
quadrature points. By taking $x=\zeta_{j}^{\mathrm{DE}}$ in Eq. (22) we get the following system of linear equations with unknowns $v_{j}^{\mathrm{DE}}(j=-N, \ldots, N)$ :

$$
v_{j}^{\mathrm{DE}}+h \sum_{i=-N}^{N} K_{1}\left(\zeta_{j}^{\mathrm{DE}}, \zeta_{i}^{\mathrm{DE}}\right)\left\{\phi^{\mathrm{DE}}\right\}^{\prime}(i h) J(i, h)(j h) v_{i}^{\mathrm{DE}}=f_{1}\left(\zeta_{j}^{\mathrm{DE}}\right) .
$$

Note that $\left\{\phi^{\mathrm{DE}}\right\}^{-1}\left(\zeta_{j}^{\mathrm{DE}}\right)=j h$, so $J(i, h)\left(\left\{\phi^{\mathrm{DE}}\right\}^{-1}\left(\zeta_{j}^{\mathrm{DE}}\right)\right)=J(i, h)(j h)$, and $h$ is given by formula (9). So, we obtain an approximate solution $v_{j}^{\mathrm{DE}}(j=-N, \ldots, N)$ of the linear system Eq. (23), which can be easily solved. Equation (23) can be written in the following discrete DE operator equation:

$$
\left(I_{N}+K_{N}^{\mathrm{DE}}\right) v_{N}^{\mathrm{DE}}=f_{N}^{\mathrm{DE}}
$$

Then, the approximate solution $v_{N}^{\mathrm{DE}}(x)$ at an arbitrary point $x$ of Eq. (14) can be expressed as

$$
v_{N}^{\mathrm{DE}}(x)=f_{1}(x)-h \sum_{i=-N}^{N} K_{1}\left(x, \zeta_{i}^{\mathrm{DE}}\right)\left\{\phi^{\mathrm{DE}}\right\}^{\prime}(i h) J(i, h)\left(\left\{\phi^{\mathrm{DE}}\right\}^{-1}(x)\right) v_{i}^{\mathrm{DE}}
$$

Indeed, $v_{N}^{\mathrm{DE}}(x)$ is also an approximate solution of Eq. (12). From formula (11) we get an approximate solution $u_{N}^{\mathrm{DE}}(x)$ for Eq. (1) of the form

$$
u_{N}^{\mathrm{DE}}(x)=H^{-1}\left(v_{N}^{\mathrm{DE}}(x)\right), \quad x \in[a, b] .
$$

\section{Convergence analysis for numerical method}

Throughout this section, we provide a convergence analysis of the associated SE and DE Sinc Nyström methods. Let us first consider the SE case. Tomoaki Okayama and his coauthors have given the theoretical analysis of Sinc Nyström methods for linear Volterra equation in [22] by utilizing error estimates with explicit constants for Sinc quadrature. They display that approximate solutions have exponential convergence order.

Theorem 4.1 Let $f^{\prime} \in \operatorname{Hol}\left(\phi^{\mathrm{SE}}\left(\mathscr{D}_{d}\right)\right)$, $\frac{\partial K(x, \cdot)}{\partial x} Q(\cdot) \in C[a, b], \frac{\partial K(\cdot, t)}{\partial t} Q(t) \in C[a, b]$ for all $x, t \in$ $[a, b]$, and $1 / K(t, t) \in C[a, b]$. Then, there exist $N_{0} \in \mathbb{Z}^{+}$and a constant $C$ such that for $N>N_{0}$, the discrete coefficient matrix $I_{N}+K_{N}^{\mathrm{SE}}$ is invertible, and

$$
\max _{a \leq x \leq b}\left|v(x)-v_{N}^{\mathrm{SE}}(x)\right| \leq C e^{-\sqrt{\pi d \alpha N}}
$$

Proof We refer to [22].

Theorem 4.2 Let the assumptions in Theorem 4.2 be satisfied. Then there exist constants $C_{1}$ and $C_{2}$ independent of $N$ such that

$$
\left\|I_{N}+K_{N}^{\mathrm{SE}}\right\|_{\infty} \leq C_{1}, \quad\left\|\left(I_{N}+K_{N}^{\mathrm{SE}}\right)^{-1}\right\|_{\infty} \leq C_{2}
$$

Proof We refer to [22].

According to these results, we can give an error analysis of the nonlinear Volterra integral equations of the first kind. 
Theorem 4.3 Let the assumptions in Theorem 4.2 be satisfied, and let the inverse function of $H(v(t))$ satisfy the Lipschitz condition with respect to $v$ of constant $L>0$, that is,

$$
\left|H^{-1}\left(v_{1}\right)-H^{-1}\left(v_{2}\right)\right| \leq L\left|v_{1}-v_{2}\right|
$$

Then there exists a constant $C_{0}$ independent of $N$ such that

$$
\max _{a \leq x \leq b}\left|u(x)-u_{N}^{\mathrm{SE}}(x)\right| \leq C_{0} e^{-\sqrt{\pi d \alpha N}} .
$$

Proof Combining Theorem 4.1 and known conditions, we have

$$
\begin{aligned}
\max _{a \leq x \leq b}\left|u(x)-u_{N}^{\mathrm{SE}}(x)\right| & =\max _{a \leq x \leq b}\left|H^{-1}(v(x))-H^{-1}\left(v_{N}^{\mathrm{SE}}(x)\right)\right| \\
& \leq L\left|v(x)-v_{N}^{\mathrm{SE}}(x)\right| \\
& \leq L C e^{-\sqrt{\pi d \alpha N}} \\
& =C_{0} e^{-\sqrt{\pi d \alpha N}} .
\end{aligned}
$$

The proof of the theorem is completed.

Next, we take into account the error analysis of DE case for Eq. (1). The proof is similar to that in the SE case, so we only state the results.

Theorem 4.4 Let $f^{\prime} \in \operatorname{Hol}\left(\phi^{\mathrm{DE}}\left(\mathscr{D}_{d}\right)\right), \frac{\partial K(x, \cdot)}{\partial x} Q(\cdot) \in C[a, b], \frac{\partial K(\cdot, t)}{\partial t} Q(t) \in C[a, b]$ for all $x, t \in$ $[a, b]$, and $1 / K(t, t) \in C[a, b]$. Then, there exist $N_{0} \in \mathbb{Z}^{+}$and a constant $C$ such that for $N>N_{0}$, the discrete coefficient matrix $I_{N}+K_{N}^{\mathrm{DE}}$ is invertible, and

$$
\max _{a \leq x \leq b}\left|v(x)-v_{N}^{\mathrm{DE}}(x)\right| \leq C \frac{\log (2 d N / \alpha)}{N} e^{\frac{-\pi d N}{\log (2 d N / \alpha)}}
$$

Theorem 4.5 Let the assumptions in Theorem 4.3 be satisfied. Then there exist constants $C_{1}$ and $C_{2}$ independent of $N$ such that

$$
\left\|I_{N}+K_{N}^{\mathrm{DE}}\right\|_{\infty} \leq C_{1}, \quad\left\|\left(I_{N}+K_{N}^{\mathrm{DE}}\right)^{-1}\right\|_{\infty} \leq C_{2} .
$$

Theorem 4.6 Let the assumptions in Theorem 4.3 be satisfied, and let the inverse function $H^{-1}(v(t))$ satisfy the Lipschitz condition with respect to $v$ of constant $L>0$, that is,

$$
\left|H^{-1}\left(v_{1}\right)-H^{-1}\left(v_{2}\right)\right| \leq L\left|v_{1}-v_{2}\right|
$$

Then there exists a constant $C_{0}$ independent of $N$ such that

$$
\max _{a \leq x \leq b}\left|u(x)-u_{N}^{\mathrm{DE}}(x)\right| \leq C_{0} \frac{\log (2 d N / \alpha)}{N} e^{\frac{-\pi d N}{\log (2 d N / \alpha)}} .
$$

Remark 1 Here, the values of $C_{0}$ in formulas (30) and (34) are different. In addition, the convergence rate of the approximate solution in (1) and (12) are consistent while the inverse function of $H(u(t))$ satisfies the Lipschitz condition. We can get the same conclusion 
for the DE case. Further, the convergence speed of the DE Sinc Nyström method is much faster than that of the SE Sinc Nyström method.

Remark 2 Theorem 4.2 and Theorem 4.5 suggest that the condition numbers of the matrices $I_{N}+K_{N}^{\mathrm{SE}}$ and $I_{N}+K_{N}^{\mathrm{DE}}$ are uniformly bounded under the infinity norm.

\section{Numerical examples}

In this section, several numerical examples are provided to illustrate the effectiveness and accuracy of the SE and DE Sinc Nyström methods, and all experiments are implemented using MATLAB. For the SE Sinc Nyström method, we choose $d=3.14$ and $\alpha=1$ in formula (6). For the DE Sinc Nyström method, we choose $d=1.57$ and $\alpha=1 / 2$ in formula (9). In the following numerical examples, in order to observe the convergence behavior and validate the theoretical results, we solve each equation for several values of $N$. Specifically, we select $N=2,4,8,16, \ldots$ and define

$$
\begin{aligned}
& \text { Error }_{v(x)}=\max \left\{\left|v\left(x_{i}\right)-v_{N}\left(x_{i}\right)\right|: x_{i}=\frac{i}{100}, i=0,1, \ldots, 100\right\}, \\
& \operatorname{Error}_{u(x)}=\max \left\{\left|u\left(x_{i}\right)-u_{N}\left(x_{i}\right)\right|: x_{i}=\frac{i}{100}, i=0,1, \ldots, 100\right\}, \\
& \rho_{N}=\log _{2}\left(\frac{\operatorname{Error}_{u(x)}^{i}}{\operatorname{Error}_{u(x)}^{(i+1)}}\right), \quad \text { Cond }=\left\|I_{N}+K_{N}\right\|_{\infty}\left\|\left(I_{N}+K_{N}\right)^{-1}\right\|_{\infty},
\end{aligned}
$$

where $\operatorname{Error}_{v(x)}$ and $\operatorname{Error}_{u(x)}$ are the maximum errors corresponding to the grid points for Eq. (12) and Eq. (1), respectively. In addition, $v_{N}$ and $u_{N}$ stand for $v_{N}^{\mathrm{SE}}$ and $u_{N}^{\mathrm{SE}}$ or $v_{N}^{\mathrm{DE}}$ and $u_{N}^{\mathrm{DE}}$, respectively, and $\rho_{N}$ denotes the convergence rate of the presented methods for Eq. (1). In formula (37), Error ${ }_{u(x)}^{i}$ represents the $\operatorname{Error}_{u(x)}$ in the $(i+1)$ th row of the following tables, and Cond denotes the condition number of matrix $I_{N}+K_{N}^{\mathrm{SE}}$ or $I_{N}+K_{N}^{\mathrm{DE}}$.

Example 1 Consider the following nonlinear Volterra integral equation of the first kind:

$$
\int_{0}^{x} e^{(x-t)} u^{2}(t) d t=e^{2 x}-e^{x}, \quad x \in[0,1]
$$

with the exact solution $u(x)=e^{x}$. This equation is converted to a linear Volterra integral equation of the second kind by means of $u^{2}(t)=v(t)$. From the relation $u(t)=H^{-1}(v(t))=$ $\sqrt{v(t)}, v \in\left[1, e^{2}\right]$, an approximate solution of this equation is gained.

Since for all $v_{1}, v_{2} \in\left[1, e^{2}\right],\left|u_{1}-u_{2}\right|=\left|H^{-1}\left(v_{1}\right)-H^{-1}\left(v_{2}\right)\right|=\left|\sqrt{v_{1}}-\sqrt{v_{2}}\right|=\left|\frac{v_{1}-v_{2}}{\sqrt{v_{1}}+\sqrt{v_{2}}}\right| \leq$ $\frac{1}{2}\left|v_{1}-v_{2}\right|$, we have that $H^{-1}(v(t))$ satisfies the Lipschitz condition with respect to $v$. The numerical results are shown in Tables 1 and 2 , which verify the conclusions of the above

Table 1 The numerical results of the SE Sinc Nyström method for Example 1

\begin{tabular}{rllll}
\hline $\boldsymbol{N}$ & Error $_{\boldsymbol{v}(\boldsymbol{x})}$ & Error $_{\boldsymbol{u}(\boldsymbol{x})}$ & $\boldsymbol{\rho}_{\boldsymbol{N}}$ & Cond \\
\hline 4 & $9.203 \mathrm{e}-003$ & $2.442 \mathrm{e}-003$ & $*$ & 5.403 \\
8 & $9.862 \mathrm{e}-004$ & $2.329 \mathrm{e}-004$ & 3.390 & 5.434 \\
16 & $3.027 \mathrm{e}-005$ & $7.625 \mathrm{e}-006$ & 4.933 & 5.437 \\
32 & $2.164 \mathrm{e}-007$ & $4.670 \mathrm{e}-008$ & 7.351 & 5.437 \\
64 & $1.536 \mathrm{e}-010$ & $3.001 \mathrm{e}-011$ & 10.604 & 5.437 \\
\hline
\end{tabular}


Table 2 The numerical results of the DE Sinc Nyström method for Example 1

\begin{tabular}{rllll}
\hline $\boldsymbol{N}$ & Error $_{\boldsymbol{v}(\boldsymbol{x})}$ & Error $_{\boldsymbol{u}(\boldsymbol{x})}$ & \multicolumn{1}{c}{$\boldsymbol{\rho}_{\boldsymbol{N}}$} & Cond \\
\hline 4 & $8.238 \mathrm{e}-002$ & $1.887 \mathrm{e}-002$ & $*$ & 5.500 \\
8 & $5.987 \mathrm{e}-003$ & $1.305 \mathrm{e}-003$ & 3.854 & 5.438 \\
16 & $1.871 \mathrm{e}-005$ & $3.476 \mathrm{e}-006$ & 8.553 & 5.437 \\
32 & $9.852 \mathrm{e}-010$ & $2.360 \mathrm{e}-010$ & 13.846 & 5.437 \\
64 & $3.553 \mathrm{e}-015$ & $8.882 \mathrm{e}-016$ & 18.020 & 5.437 \\
\hline
\end{tabular}
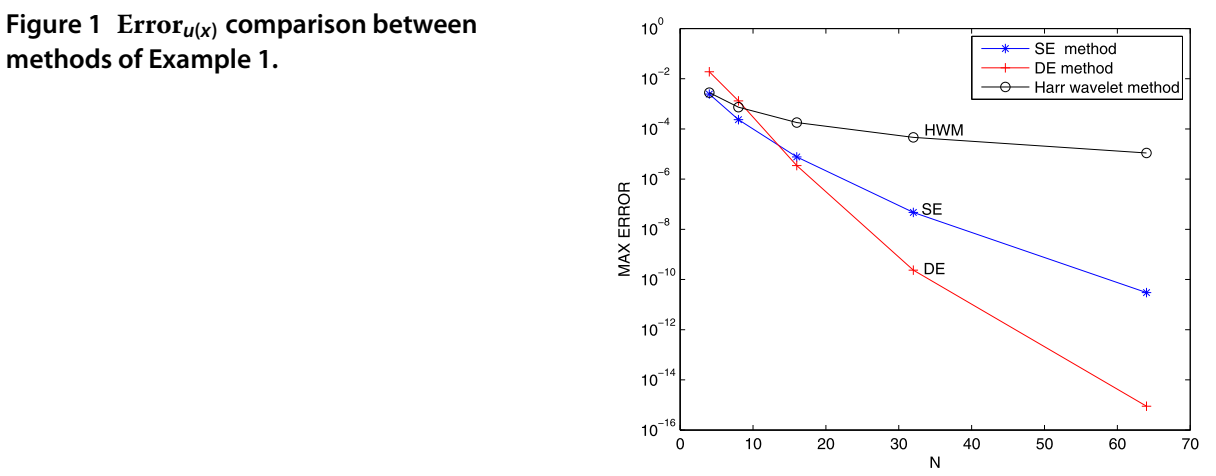

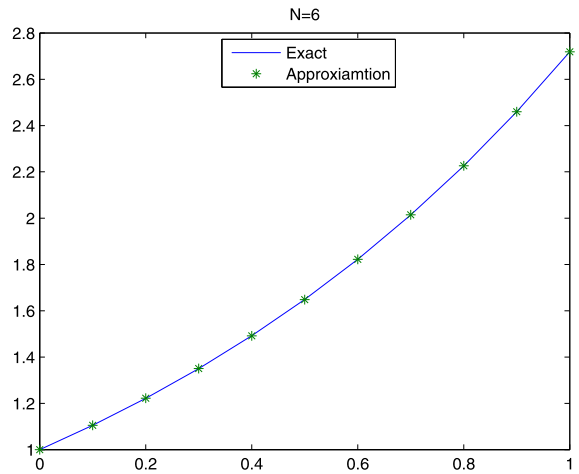

(a) SE Sinc Nyström method

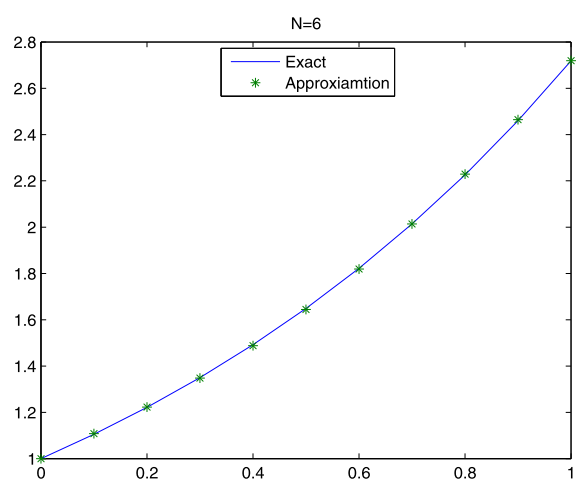

(b) DE Sinc Nyström method

Figure 2 The numerical results and exact solutions of Example 1.

theorems. The results present the exponential convergence rate of described methods, and the condition numbers of the matrices $I_{N}+K_{N}^{\mathrm{SE}}$ and $I_{N}+K_{N}^{\mathrm{DE}}$ are uniformly bounded with infinity norm. By increasing the value of $N$ the error decreases. As anticipated, Tables 1 and 2 illustrate that the convergence speed of the DE Sinc Nyström method is much faster than that of the SE Sinc Nyström method. Figure 1 represents the numerical results of the SE Sinc Nyström method, DE Sinc Nyström method, and Haar wavelet method [14]. When the value of $N$ is small, the Haar wavelet method is more efficient than the DE Sinc Nyström method. Yet, it is displayed that the convergence rate of present methods is much faster than that of the Haar wavelet method. In fact, the convergence order of the error for Haar wavelet method is $O\left(\frac{1}{m}\right)$. In Figure 2, the values of exact solution and approximate solution with $N=6$ for our methods are provided. The figure shows the accuracy of the proposed methods. 
Example 2 Consider the following nonlinear Volterra integral equation of the first kind:

$$
\int_{0}^{x} e^{(x-t)} \ln (u(t)) d t=e^{x}-x-1, \quad x \in[0,1]
$$

with the exact solution $u(x)=e^{x}$. This equation is converted to a linear Volterra integral equation of the second kind by employing $\ln (u(t))=v(t)$. According to the identity $u(t)=$ $e^{v(t)}$, the approximate solution of this equation is achieved.

Based on the Lagrange theorem, $u=H^{-1}(v(t))=e^{v}$ satisfies the Lipschitz condition with respect to $v$. The numerical results of Tables 3 and 4 present the exponential convergence rate of the presented methods. In addition, the condition number of the matrices in each row is small and bound. The tables demonstrate that the convergence speed of the DE Sinc Nyström method is much faster than that of the SE Sinc Nyström method, as predicted. Figure 3 displays the numerical results obtained from the SE Nyström Sinc method, DE Sinc Nyström method, and Haar wavelet method [14]. When the value of $N$ is small, the Haar wavelet method is better than the DE Sinc Nyström method. But it is clear that the convergence speed of the proposed methods is much quicker than the Haar wavelet method. Figure 4 shows the curves of exact solution and approximate solution with $N=6$ of the proposed methods. The results approve the efficiency of this method for solving these problems.

Table 3 The numerical results of the SE Sinc Nyström method for Example 2

\begin{tabular}{rllll}
\hline $\boldsymbol{N}$ & Error $_{\boldsymbol{v}(\boldsymbol{x})}$ & Error $_{\boldsymbol{u}(\boldsymbol{x})}$ & $\boldsymbol{\rho}_{\boldsymbol{N}}$ & Cond \\
\hline 4 & $1.701 \mathrm{e}-003$ & $4.167 \mathrm{e}-003$ & $*$ & 5.403 \\
8 & $1.766 \mathrm{e}-004$ & $4.658 \mathrm{e}-004$ & 3.161 & 5.434 \\
16 & $9.051 \mathrm{e}-006$ & $2.365 \mathrm{e}-005$ & 4.301 & 5.437 \\
32 & $1.039 \mathrm{e}-007$ & $2.602 \mathrm{e}-007$ & 6.505 & 5.437 \\
64 & $1.360 \mathrm{e}-010$ & $3.175 \mathrm{e}-010$ & 9.678 & 5.437 \\
\hline
\end{tabular}

Table 4 The numerical results of the DE Sinc Nyström method for Example 2

\begin{tabular}{rllll}
\hline $\boldsymbol{N}$ & Error $_{\boldsymbol{v}(\boldsymbol{x})}$ & Error $_{\boldsymbol{u}(\boldsymbol{x})}$ & \multicolumn{1}{c}{$\boldsymbol{\rho}_{\boldsymbol{N}}$} & Cond \\
\hline 4 & $1.541 \mathrm{e}-002$ & $2.591 \mathrm{e}-002$ & $*$ & 5.500 \\
8 & $8.811 \mathrm{e}-004$ & $2.325 \mathrm{e}-003$ & 3.478 & 5.438 \\
16 & $1.308 \mathrm{e}-005$ & $3.266 \mathrm{e}-005$ & 6.154 & 5.437 \\
32 & $6.183 \mathrm{e}-010$ & $1.139 \mathrm{e}-009$ & 14.817 & 5.437 \\
64 & $5.551 \mathrm{e}-016$ & $8.882 \mathrm{e}-016$ & 20.291 & 5.437 \\
\hline
\end{tabular}

Figure 3 Error $_{u(x)}$ comparison between methods of Example 2.

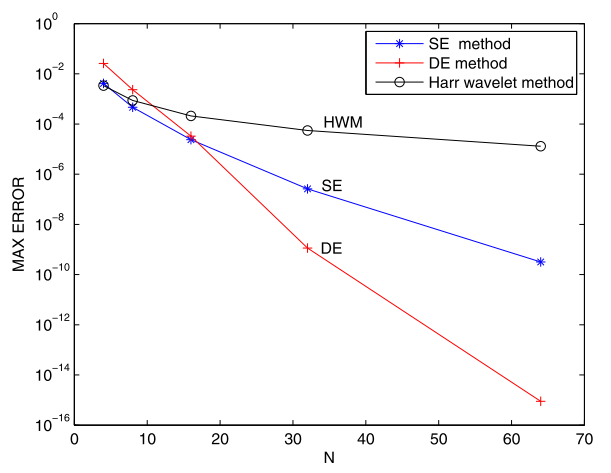




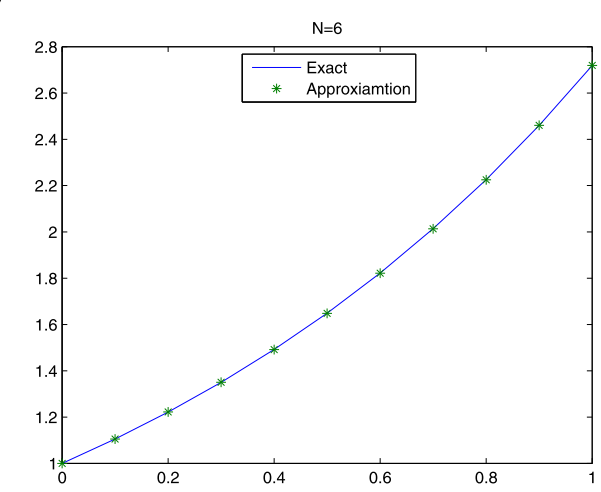

(a) SE Sinc Nyström method

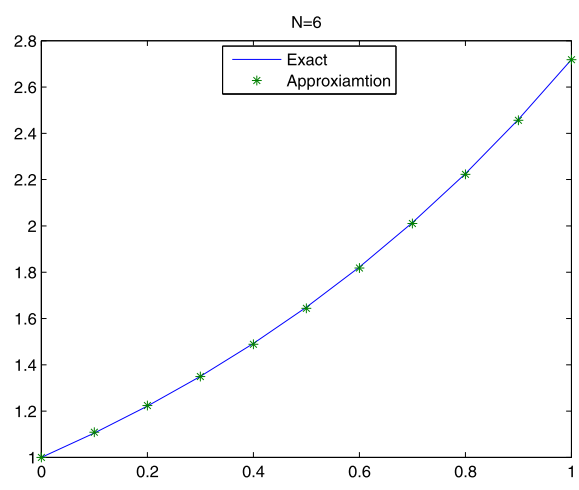

(b) DE Sinc Nyström method

Figure 4 The numerical results and exact solutions of Example 2.

Table 5 The numerical results of the SE Sinc Nyström method for Example 3

\begin{tabular}{rllll}
\hline $\boldsymbol{N}$ & Error $_{\boldsymbol{v}(\boldsymbol{x})}$ & Error $_{\boldsymbol{u}(\boldsymbol{x})}$ & $\boldsymbol{\rho}_{\boldsymbol{N}}$ & Cond \\
\hline 4 & $3.565 \mathrm{e}-003$ & $5.677 \mathrm{e}-002$ & $*$ & 2.814 \\
8 & $3.096 \mathrm{e}-004$ & $4.437 \mathrm{e}-003$ & 3.678 & 2.823 \\
16 & $1.388 \mathrm{e}-005$ & $2.006 \mathrm{e}-004$ & 4.467 & 2.824 \\
32 & $1.547 \mathrm{e}-007$ & $1.211 \mathrm{e}-006$ & 7.372 & 2.824 \\
64 & $2.502 \mathrm{e}-010$ & $6.334 \mathrm{e}-010$ & 10.901 & 2.824 \\
\hline
\end{tabular}

Table 6 The numerical results of the DE Sinc Nyström method for Example 3

\begin{tabular}{rllll}
\hline $\boldsymbol{N}$ & Error $_{\boldsymbol{v}(\boldsymbol{x})}$ & Error $_{\boldsymbol{u}(\boldsymbol{x})}$ & $\boldsymbol{\rho}_{\boldsymbol{N}}$ & Cond \\
\hline 4 & $3.044 \mathrm{e}-002$ & $9.255 \mathrm{e}-002$ & $*$ & 2.866 \\
8 & $1.686 \mathrm{e}-003$ & $3.949 \mathrm{e}-003$ & 4.551 & 2.825 \\
16 & $1.439 \mathrm{e}-005$ & $8.880 \mathrm{e}-005$ & 5.475 & 2.824 \\
32 & $2.405 \mathrm{e}-009$ & $4.197 \mathrm{e}-008$ & 11.047 & 2.824 \\
64 & $7.772 \mathrm{e}-016$ & $8.295 \mathrm{e}-015$ & 22.271 & 2.824 \\
\hline
\end{tabular}

Example 3 Consider the following nonlinear Volterra integral equation of the first kind:

$$
\int_{0}^{x}(\sin (x-t)+1) \cos (u(t)) d t=\frac{x \sin x}{2}+\sin x, \quad x \in[0,1]
$$

with the exact solution $u(x)=x$. When $\cos (u(t))=v(t)$ is utilized, this equation is transformed into a linear Volterra integral equation of the second kind. On the basis of $u(t)=$ $\arccos (v(t))$, the approximate solution of this equation is achieved.

Similarly, $u=H^{-1}(v(t))=\arccos (v), v \in[0, \cos (1)]$, fulfills the Lipschitz condition with respect to $v$ according to the Lagrange theorem, Tables 5 and 6 present the exponential convergence rate of described methods and show that the condition number of the discrete coefficient matrices is uniformly bounded under infinity norm. As expected, the tables show that the convergence rate of the DE Sinc Nyström method is much faster than that of the SE Nyström method. Compared with the SE, DE Sinc Nyström method, and the Haar wavelet method in Figure 5, the Haar wavelet method is more accurate than the SE Sinc Nyström method and DE Sinc Nyström method when the integer $N$ is small. However, the proposed algorithms have a faster convergence speed than the Haar wavelet method [14], 


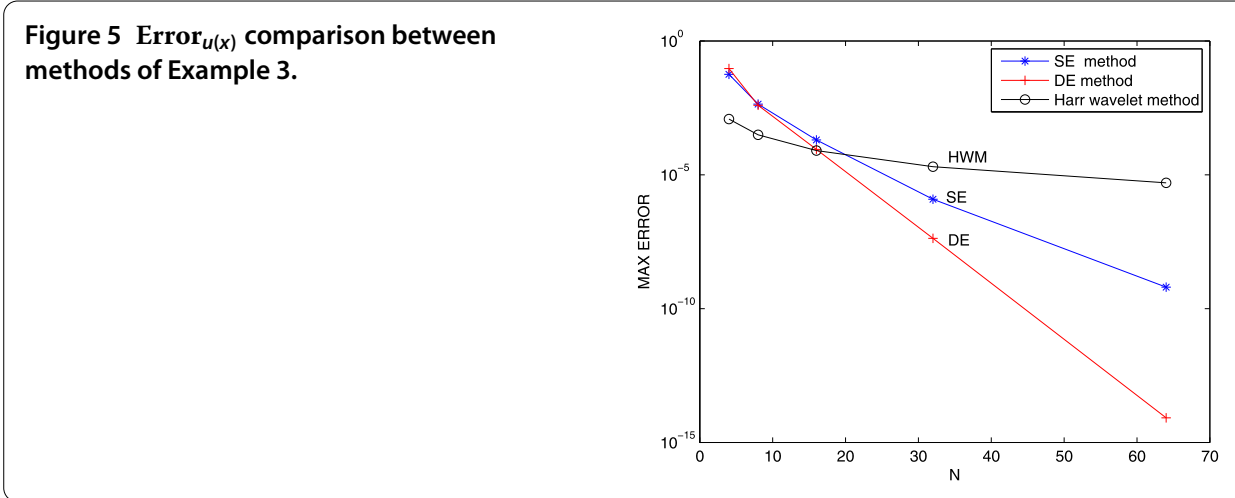

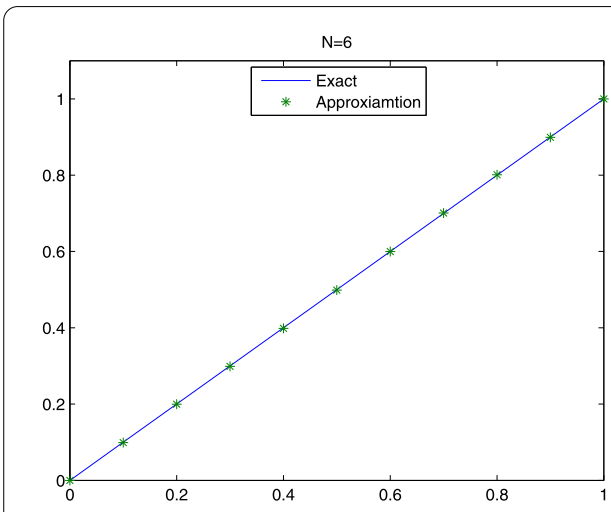

(a) SE Sinc Nyström method

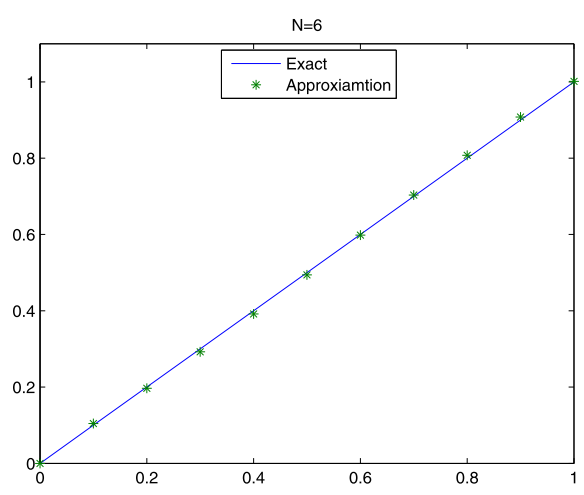

(b) DE Sinc Nyström method

Figure 6 The numerical results and exact solutions of Example 3.

Table 7 The numerical results of the SE Sinc Nyström method for Example 4

\begin{tabular}{rllll}
\hline $\boldsymbol{N}$ & Error $_{\boldsymbol{v}(\boldsymbol{x})}$ & Error $_{\boldsymbol{u}(\boldsymbol{x})}$ & $\boldsymbol{\rho}_{\boldsymbol{N}}$ & Cond \\
\hline 4 & $4.079 \mathrm{e}-003$ & $3.518 \mathrm{e}-003$ & $*$ & 2.330 \\
8 & $3.576 \mathrm{e}-004$ & $2.783 \mathrm{e}-004$ & 3.660 & 2.337 \\
16 & $9.208 \mathrm{e}-006$ & $1.611 \mathrm{e}-005$ & 4.111 & 2.338 \\
32 & $9.411 \mathrm{e}-008$ & $2.283 \mathrm{e}-007$ & 6.140 & 2.338 \\
64 & $1.498 \mathrm{e}-010$ & $3.041 \mathrm{e}-010$ & 9.552 & 2.338 \\
\hline
\end{tabular}

so they are very considerable. The values of the exact solution and approximate solution with $N=6$ for our methods are presented in Figure 6 .

Example 4 Consider the following nonlinear Volterra integral equation of the first kind:

$$
\int_{0}^{x} e^{x+t} u^{\frac{3}{2}}(t) d t=(x-1) e^{2 x}-e^{x}, \quad x \in[0,1]
$$

with the exact solution $u(x)=x^{\frac{2}{3}}$. When the $u^{\frac{3}{2}}(t)=v(t)$ is utilized, this equation is transformed into a linear Volterra integral equation of the second kind. On the basis of $u(t)=v^{\frac{2}{3}}(t)$, the approximate solution of this equation is achieved.

Here $u=H^{-1}(v(t))=v^{\frac{2}{3}}, v \in[0,1]$, does not satisfy the Lipschitz condition with respect to $v$. However, Tables 7 and 8 show that the results are in agreement with the previous 
Table 8 The numerical results of the DE Sinc Nyström method for Example 4

\begin{tabular}{rllll}
\hline $\boldsymbol{N}$ & Error $_{\boldsymbol{v}(\boldsymbol{x})}$ & Error $_{\boldsymbol{u}(\boldsymbol{x})}$ & $\boldsymbol{\rho}_{\boldsymbol{N}}$ & Cond \\
\hline 4 & $3.100 \mathrm{e}-002$ & $2.895 \mathrm{e}-002$ & $*$ & 2.339 \\
8 & $1.912 \mathrm{e}-003$ & $1.362 \mathrm{e}-004$ & 4.410 & 2.338 \\
16 & $1.420 \mathrm{e}-005$ & $4.278 \mathrm{e}-005$ & 4.992 & 2.338 \\
32 & $8.818 \mathrm{e}-009$ & $9.879 \mathrm{e}-0010$ & 15.402 & 2.338 \\
64 & $2.220 \mathrm{e}-016$ & $3.053 \mathrm{e}-016$ & 21.626 & 2.338 \\
\hline
\end{tabular}

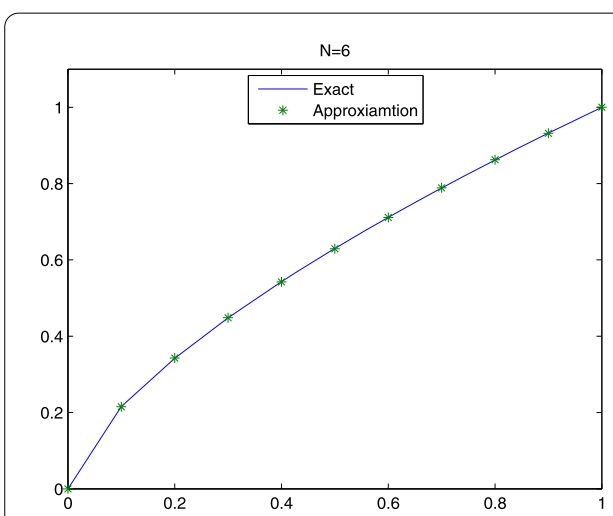

(a) SE Sinc Nyström method

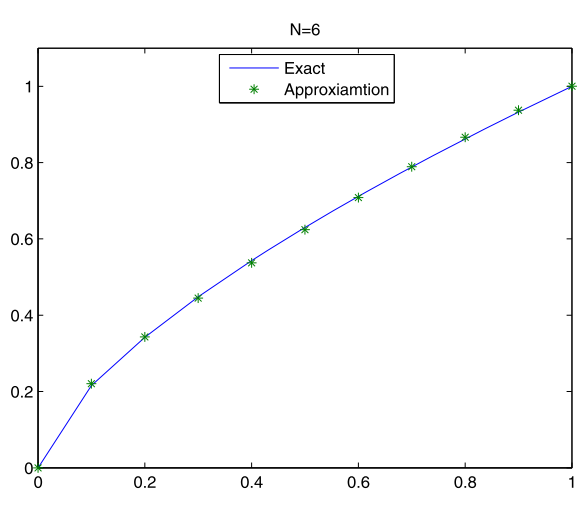

(b) DE Sinc Nyström method

Figure 7 The numerical results and exact solutions of Example 4.

examples. In Figure 7, the curves of the exact solution and the approximated solution with $N=6$ for the proposed methods are plotted. This example suggests that the conditions of Theorem 4.3 and Theorem 4.6 can be weaken, and we can draw the same conclusions.

\section{Conclusion}

In the present study, the SE and DE Nyström method are presented by converting nonlinear Volterra integral equations of the first kind into linear Volterra integral equations of the second kind. The proposed methods are stable and avoid the ill-conditioning and nonlinear iteration problems. The condition numbers have good reliability and efficiency. Numerical results are in agreement with the theoretical analysis. It is obvious that the convergence rate of the approximate solutions are exponential when the inverse function of $H(u(t))$ satisfies the Lipschitz condition. In future work, we will utilize the proposed methods to deal with the general nonlinear Volterra integral equations of the first kind and nonlinear Volterra integral equation systems of the first kind.

The authors declare that they have no competing interests.

\section{Authors' contributions}

The authors declare that the study was realized in collaboration with the same responsibility. All authors read and approved the final manuscript.

\section{Author details}

'School of Mathematical Sciences, University of Electronic Science and Technology of China, Chengdu, Sichuan 611731, P.R. China. ${ }^{2}$ School of Automation Engineering, University of Electronic Science and Technology of China, Chengdu, Sichuan 611731, P.R. China. 


\section{Acknowledgements}

The authors are very grateful to the referees for their detailed comments and valuable suggestions, which greatly improved the manuscript. This work was partially supported by the financial support from National Natural Science Foundation of China (Grant No. 11371079).

Received: 19 January 2016 Accepted: 28 April 2016 Published online: 08 June 2016

\section{References}

1. Babolian, E, Delves, LM: An augmented Galerkin method for first kind Fredholm equations. IMA J. Appl. Math. 24, 157-174 (1979)

2. Delves, LM, Mohamed, J: Computational Methods for Integral Equations. Cambridge University Press, Cambridge (1988)

3. Lamm, PK: Approximation of ill-posed Volterra problems via predictor-corrector regularization methods. SIAM J. Appl. Math. 56(2), 524-541 (1996)

4. Lamm, PK, Eldén, L: Numerical solution of first kind Volterra equations by sequential Tikhonov regularization. SIAM J. Numer. Anal. 34(4), 1432-1450 (1997)

5. Lamm, PK: Solution of ill-posed Volterra equations via variable-smoothing Tikhonov regularization. In: Inverse Problems in Geophysical Applications (Yosemite, CA, 1995), pp. 92-108. SIAM, Philadelphia (1997)

6. Babolian, E, Masouri, Z: Direct method to solve Volterra integral equation of the first kind using operational matrix with block-pulse functions. J. Comput. Appl. Math. 220(1), 51-57 (2008)

7. Maleknejad, K, Rahimi, B: Modification of block pulse functions and their application to solve numerically Volterra integral equation of the first kind. Commun. Nonlinear Sci. Numer. Simul. 16(6), 2469-2477 (2011)

8. Maleknejad, K, Mollapourasl, R, Alizadeh, M: Numerical solution of Volterra type integral equation of the first kind with wavelet basis. Appl. Math. Comput. 194(2), 400-405 (2007)

9. Masouri, Z, Babolian, E, Hatamzadeh-Varmazyar, S: An expansion-iterative method for numerically solving Volterra integral equation of the first kind. Comput. Math. Appl. 59(4), 1491-1499 (2010)

10. Babolian, E, Shamloo, AS: Numerical solution of Volterra integral and integro-differential equations of convolution type by using operational matrices of piecewise constant orthogonal functions. J. Comput. Appl. Math. 214(2), 495-508 (2008)

11. Biazar, J, Eslami, M, Aminikhah, H: Application of homotopy perturbation method for systems of Volterra integral equations of the first kind. Chaos Solitons Fractals 42(5), 3020-3026 (2009)

12. Biazar, J, Babolian, E, Islam, R: Solution of a system of Volterra integral equations of the first kind by Adomian method. Appl. Math. Comput. 139(2), 249-258 (2003)

13. Khan, N, Hashmi, M, lqbal, S, Mahmood, T: Optimal homotopy asymptotic method for solving Volterra integral equation of first kind. Alex. Eng. J. 53(3), 751-755 (2014)

14. Singh, I, Kumar, S: Haar wavelet method for some nonlinear Volterra integral equations of the first kind. J. Comput. Appl. Math. 292, 541-552 (2016)

15. Muhammad, M, Mori, M: Double exponential formulas for numerical indefinite integration. J. Comput. Appl. Math $161(2), 431-448(2003)$

16. Haber, S: Two formulas for numerical indefinite integration. Math. Comput. 60(201), 279-296 (1993)

17. Bialecki, B, Stenger, F: Sinc-Nyström method for numerical solution of one-dimensional Cauchy singular integral equation given on a smooth arc in the complex plane. Math. Comput. 51(183), 133-165 (1988)

18. Muhammad, M, Nurmuhammad, A, Mori, M, Sugihara, M: Numerical solution of integral equations by means of the Sinc collocation method based on the double exponential transformation. J. Comput. Appl. Math. 177(2), 269-286 (2005)

19. Rashidinia, J, Zarebnia, M: Convergence of approximate solution of system of Fredholm integral equations. J. Math. Anal. Appl. 333(2), 1216-1227 (2007)

20. Okayama, T, Matsuo, T, Sugihara, M: Error estimates with explicit constants for Sinc approximation, Sinc quadrature and Sinc indefinite integration. Numer. Math. 124(2), 361-394 (2013)

21. Mesgarani, $\mathrm{H}$, Mollapourasl, $\mathrm{R}$ : Theoretical investigation on error analysis of Sinc approximation for mixed Volterra-Fredholm integral equation. Comput. Math. Math. Phys. 53(5), 530-539 (2013)

22. Okayama, T, Matsuo, T, Sugihara, M: Theoretical analysis of Sinc-Nyström methods for Volterra integral equations. Math. Comput. 84(293), 1189-1215 (2015)

23. Secer, A, Alkan, S, Akinlar, MA, Bayram, M: Sinc-Galerkin method for approximate solutions of fractional order boundary value problems. Bound. Value Probl. 2013, 281 (2013)

24. Maleknejad, K, Nedaiasl, K, Moradi, B: Double exponential Sinc Nyström solution of the Urysohn integral equations. In: Proceedings of the World Congress on Engineering, vol. 1 (2013)

25. Araghi, MAF, Gelian, GK: Numerical solution of nonlinear Hammerstein integral equations via sinc collocation method based on double exponential transformation. Math. Sci. 7, Article 30 (2013)

26. Stenger, F: Numerical Methods Based on Sinc and Analytic Functions. Springer, New York (2012) 